\title{
miR-375 inhibits the proliferation and invasion of glioblastoma by regulating Wnt5a
}

\author{
G. F. $\mathrm{LI}^{1}$, Y. Y. CHENG ${ }^{2}$, B. J. LI ${ }^{3}$, C. ZHANG ${ }^{2}$, X. X. ZHANG ${ }^{1}$, J. SU' ${ }^{1}$, C. WANG ${ }^{1}$, L. CHANG ${ }^{1}$, D. Z. ZHANG ${ }^{1}$, C. L. TAN ${ }^{1}$, N. WANG $^{4}, *$ \\ ${ }^{1}$ Department of Neurosurgery, ${ }^{2}$ Department of Ultrasound, ${ }^{3}$ Department of Head and Neck Surgery, Harbin Medical University Cancer Hospital, \\ Harbin, Heilongjiang, China; ${ }^{4}$ Department of Neurosurgery, The First Affiliated Hospital of Harbin Medical University, Harbin, Heilongjiang, \\ China
}

${ }^{*}$ Correspondence: wangningwsfm@163.com

Received July 14, 2018 / Accepted November 6, 2018

\begin{abstract}
The aberrant expression of microRNA-375 (miR-375) has been proven to be associated with carcinogenesis. However, the role of miR-375 in glioblastoma (GBM) remains unknown. The aim of this study was to investigate miR-375 biological functions and its molecular mechanisms in GBM cells. In this study, real-time PCR results showed that the level of miR-375 expression in GBM tissues and GBM cell lines (U87 and U251) was decreased. Using MTT assay, trans-well migration and invasion assay, we demonstrated that miR-375 overexpression significantly suppress cell proliferation, cell migration and cell invasion capacity in U87 and U251 cells. However, downregulation of miR-375 had reverse effects on cell proliferation, migration and invasion. In association analysis, dual luciferase assay, RT-PCR and western blot analysis results confirmed that miR-375 could target the 3'UTR of Wnt5a mRNA and regulate its protein expression. Further studies also showed that Wnt5a overexpression significantly reversed miR-375-mediated proliferation, migration and invasion in U87 and U251 cells. Therefore, we concluded that miR-375 inhibits proliferation and invasion of GBM by regulating Wnt5a and therefore might be a possible therapeutic agent for GBM.
\end{abstract}

Key words: miR-375, Wnt5a, proliferation, invasion, glioblastoma

Glioblastoma (GBM) is the most aggressive and common primary brain tumor in humans. In spite of the significant improvements in the GBM treatment, the prognosis of GBM remains unsatisfactory. To explore novel therapeutic strategies for GBM, it is important to investigate the mechanism underlying GBM development [1-3]. MicroRNAs (miRNAs) are a family of non-coding, single strand, small, endogenous RNA molecules that are able to mediate target gene expression at the posttranscriptional level [4-8]. More and more studies show that miRNAs participate in the regulation of various physiological and pathological processes, including migration, invasion, apoptosis, development, differentiation and proliferation, especially in carcinogenesis [5-9]. Recently, aberrant expression of miR- 375 has been proven to be associated with carcinogenesis, including gastric cancer, colorectal cancer as well as melanoma [10-12]. However, the role of miR-375 in GBM remains unknown.

In this study, we validated the expression of miR-375 in GBM samples and GBM cell lines and analyzed the miR-375 function in proliferation and invasion of GBM cells. Furthermore, we identified Wnt5a as one of the direct target genes for miR-375 to mediate GBM cell proliferation and invasion. Our results provide a better understanding of the molecular mechanism by which miR-375 regulates GBM.

\section{Materials and methods}

Patient specimens and cell lines. We obtained a total of 56 samples from the Hospital of Harbin Medical University, including 31 samples from patients with GBM and 25 normal brain samples. Approval was obtained from the Ethics Committee of the same hospital. The normal human astrocytes (NHA) and two GBM cell lines (U87 and U251) were purchased from Chinese Academy of Sciences Cell Bank. Cells were taken from liquid nitrogen and then quickly thawed in a $37^{\circ} \mathrm{C}$ water bath. After centrifugation, the cells were collected, placed into DMEM culture medium containing $10 \%$ fetal calf serum and then cultured in a $37^{\circ} \mathrm{C}$ incubator. Culture medium was replaced periodically. After the tumor cells grew to approximately $80 \%$ of the bottle bottom wall, they were digested and transferred by $0.25 \%$ trypsin and then cultured in a fresh culture bottle. 
Transfection. The negative control (NC), miR-137 inhibitor and miR-137 mimics were bought from GenePharma (GenePharma, Shanghai, China). U87 $\left(5 \times 10^{5}\right)$ and U251 $\left(5 \times 10^{5}\right)$ were seeded in $6 \mathrm{~cm}$ dishes. The NC, miR-137 inhibitor, miR-375 mimic and miR-375 mimics+ Wnt5a expressing vectors (pSUPER-Wnt5a) were inoculated in U87 and U251 using Lipofectamine 2000 (GenePharma, China) according to the manufacturer's manual. The transfection rate of the cells was observed by RT-PCR.

RT-PCR. The total RNA was extracted according to the specifications as described in the kit instruction of Trizol Reagent (Alphainnotech Company, US). The ratio of the absorption value at $260 \mathrm{~nm}$ to that at $280 \mathrm{~nm}$ was measured by the micro-spectrophotometer to evaluate the purity of RNA and the ratio scope was determined to be 1.8 2.1. To examine the miR expression, real-time qPCR was conducted using PrimeScript ${ }^{\oplus}$ miRNA RT-PCR Kit (Takara, Dalian, P.R. China) on a 7900HT Real-Time PCR System (Applied Biosystems). To examine the expression of mRNA, real-time qPCR was conducted using the standard SYBR Green RT-PCR Kit (Applied Biosystems, USA). U6 and GAPDH were used as internal references. The thermal-cycling profile: $95^{\circ} \mathrm{C}$ for 10 minutes, followed by 40 amplification cycles at $95^{\circ} \mathrm{C}$ for 30 sec and $60^{\circ} \mathrm{C}$ for 1 minute. The relative expression amounts

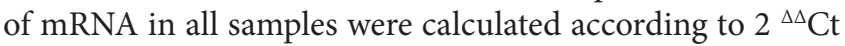
method. Each assay was carried out in triplicate and all experiments were repeated at least three times.

MicroRNA target prediction and luciferase reporter assay. TargetScan (www.targetscan.org) was used to predict the putative target genes of miR-375. U87 or U251 cells were seeded in a 24-well plate. 24 hours later, cells were co-transfected with luciferase vectors, either with the mutated Wnt5a 3'UTR reporter plasmid or the wild-type Wnt5a 3'UTR reporter plasmid together with miRNA-NC or miR-375 mimics, and the Renilla control. Luciferase activity was measured after 48 hours by using the Dual-Luciferase Reporter Assay System (Promega, USA) and normalized to Renilla luciferase activity. The experiments were conducted in triplicate and repeated three times.

Cell proliferation assay. U251 or U87 cells were incubated in 96-well plate after transfection. Then, $20 \mu \mathrm{l} \mathrm{MTT}$ was added into each well periodically and incubated at $37^{\circ} \mathrm{C}$ for $4 \mathrm{~h}$, and then liquid supernatant in the well was removed and $150 \mathrm{ml}$ DMSO was added for full dissolving. Enzyme-linked immune detector (Alphainnotech Company, U.S.A.) was used to detect the absorbance value of each well at $490 \mathrm{~nm}$. The experiments were done in triplicate and repeated three times.

Trans-well migration and invasion assays. Transwell membrane filter inserts $(8-\mu \mathrm{m}$ pore size; Costar) were pre-coated with 1-2 mg/ml Matrigel. U87 or U251 cells were transfected with NC, miR-375 mimics, miR-375 inhibitor, anti-NC, miR-375 mimics+ pSUPER and miR-375 mimics+ pSUPER-Wnt5a for 24 hours. Inferior chamber was filled with $500 \mu \mathrm{l}$ DMEM culture medium containing 10\% fetal calf serum, and upper chamber was incubated with $200 \mu \mathrm{l}$ serum-free $1 \times 10^{6}$ cell suspension. After $24 \mathrm{~h}$ culture, the cells that had invaded into inferior chamber were fixed with methanol, stained with hematoxylin and eosin and photographed under the microscope (Olympus Company, Japan). The number of cells passed through trans-well membrane in different groups was counted. The experiment was repeated for 3 times to acquire the average value. Trans-well migration assay was similar to Trans-well invasion assay but with a shorter incubation period ( $12 \mathrm{~h}$ ) and no matrigel coating. Each assay was repeated at least three times.

Western blot. Cell lysis buffer (Wuhan Zhongzhi Biotechnologies Co., Ltd., Wuhan, China) was used to extract the total cell proteins. SDS-PAGE electrophoresis was performed after the extracted total proteins were degenerated. After electrophoresis, the proteins were transferred to the prepared nitrocellulose membrane, and then nitrocellulose membrane was sealed by $5 \%$ skim milk powder for 2 hours. After washing, the first antibody was added and incubated overnight at $4{ }^{\circ} \mathrm{C}$. On the next day, PBS was used for single washing and TBST was adopted for washing for two times. Then, the second antibody marked with horseradish peroxidase was added for 1 hour incubation at room temperature. After the membrane was washed, ECL chemiluminescence was used for color developing, and X-ray film was used for developing, fixing and scanning. $\beta$-actin was used as the internal reference. The experiments were independently repeated in triplicates.

Statistics processing. Quantitative data were expressed as the mean \pm standard deviation, and SPSS19.0 software was used for statistical analysis. For comparisons between two different groups, the Student's t-test was performed. A p $<0.05$ indicates the comparison is statistically significant.

\section{Results}

MiR-375 is significantly down-regulated in GBM cell lines and tissues. To investigate the level of miR-375 expression in GBM, we first detected its expression in human GBM tissues and normal brain tissues. We also detected its expression in NHA, U87 and U251 by RT-PCR. Our results show that the level of miR-375 expression in GBM tissues is lower than in normal brain tissues (Figure 1A). Furthermore, the level of expression of miR-375 in GBM cell lines (U87 and U251) is lower than in NHA (Figure 1B). These results suggest that miR-375 might play an important role in the GBM development.

MiR-375 negatively regulates proliferation and invasion of GBM cells. To investigate the effect of miR-375 on GBM cell proliferation and invasion, the miR-375 mimics, miR-375 inhibitor or NC were transfected into the U87 and U251 cells (Figure 2A). The MTT assay showed that over-expression of miR-375 significantly suppresses proliferation of U87 and U251. In contrast, miR-375 inhibitor increased cell proliferation capacity (Figures 2B and 2C). Furthermore, trans-well migration and invasion assay demonstrated that up-regula- 
A

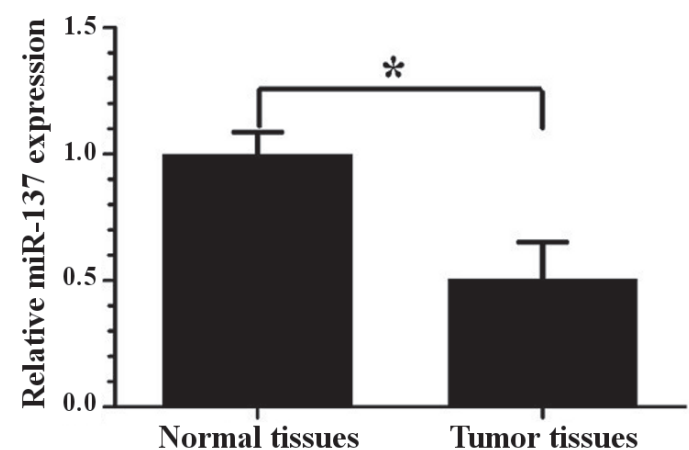

B

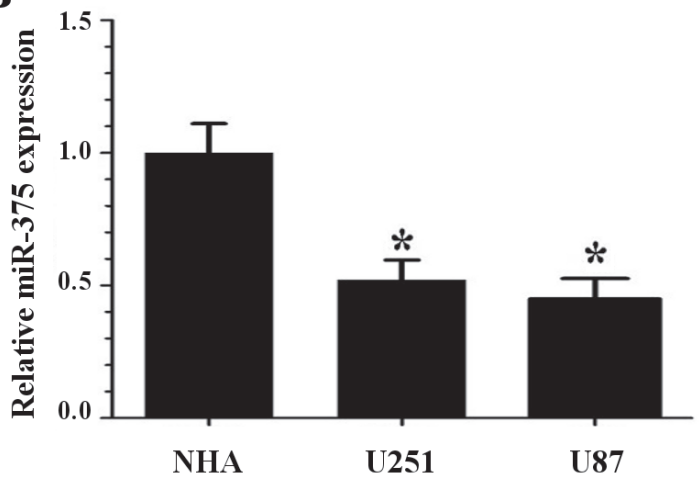

Figure 1. MiR-375 expression is decreased in GBM samples and cell lines. A) The expression of miR-375 in GBM samples and normal brain tissues. B) The levels of miR-375 expression in normal human astrocytes (NHA) and two GBM cell lines (U251 and U87) were measured by RT-PCR. ${ }^{\star}$ p $<0.05$ vs Normal or NHA.

A

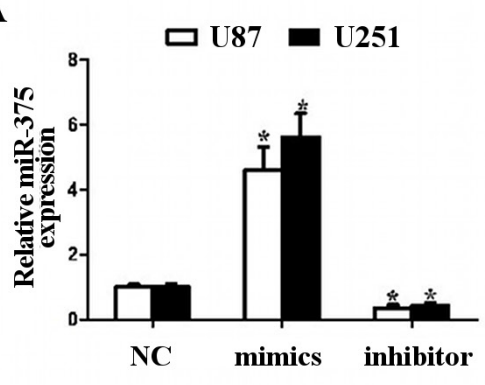

B

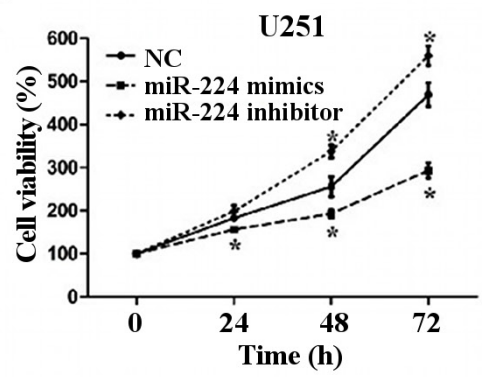

C

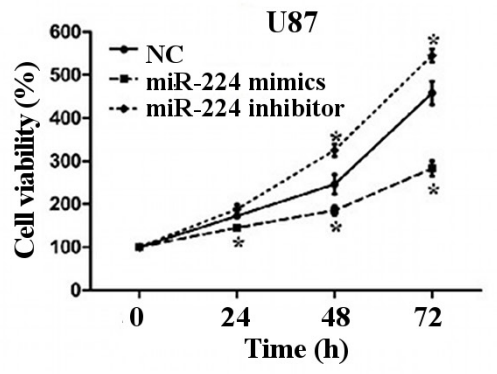

D
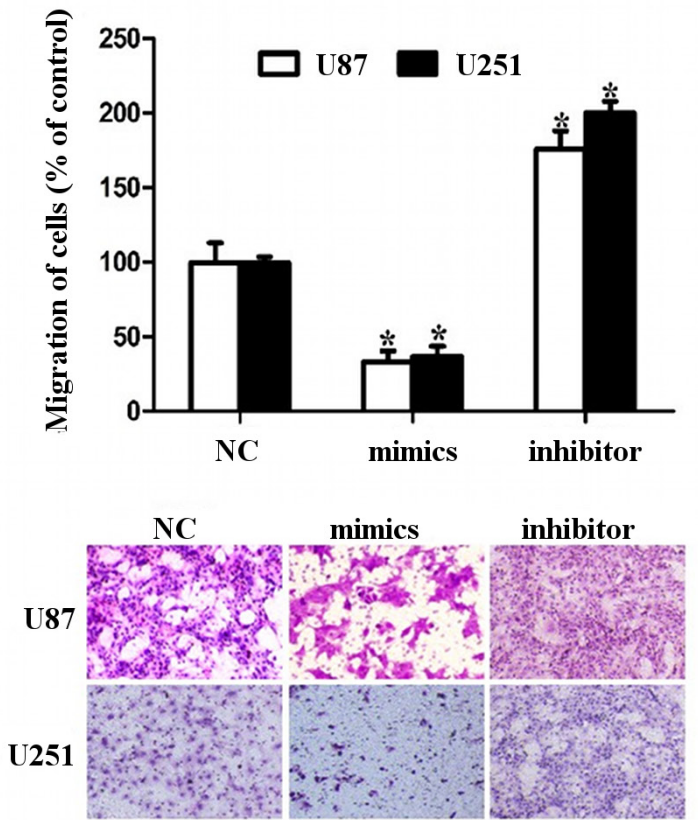

E
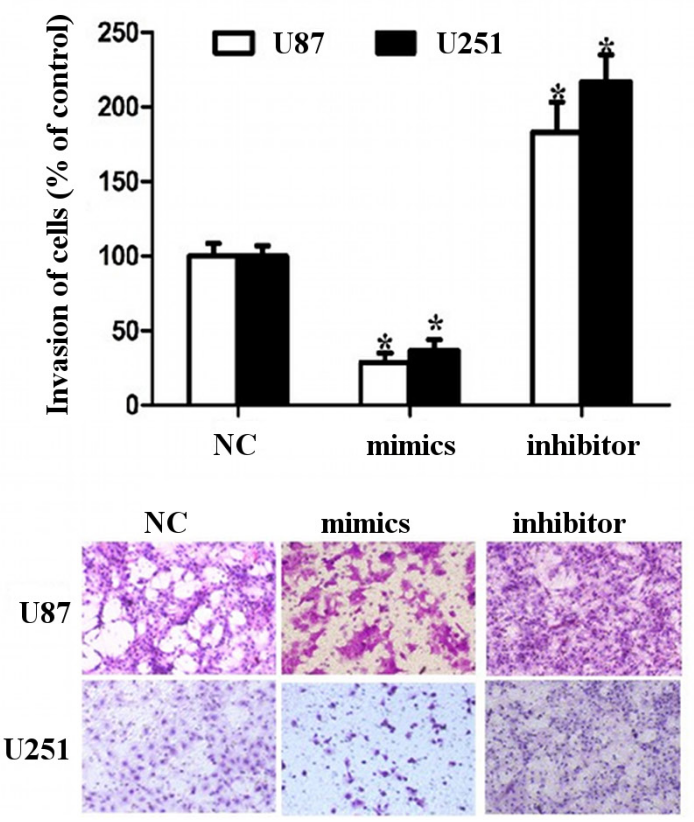

Figure 2. MiR-375 negatively influences cell proliferation and invasion in U87 and U251 cells. A) miR-375 expression in both U87 and U251 cells transfected with negative control (NC), miR-375 mimics or miR-375 inhibitor plasmid. Cell proliferation was measured by MTT assay in U87 (B) and U251 (C) cells, respectively. Trans-well migration and invasion assays were used to determine migration (D) and invasion (E) in U87 and U251 cells. ${ }^{*} \mathrm{p}<0.05$ vs NC. 
tion of miR-375 could also impair migration and invasion capability of U87 and U251 cells, but miR-375 inhibitor enhanced U87 and U251 cell migration and invasion ability (Figures 2D and 2E). These findings suggest that miR-375 may negatively regulate proliferation and invasion of GBM cells.

Wnt5a was identified as a direct target of miR-375. We found the molecular targets of miR-375 in the TargetScan database (http://www.targetscan.org), among which miR-375 had a putative binding site with the 3'-UTR of Wnt5a (Figure 3A). The wild-type Wnt5a 3'UTR reporter plasmid or mutated Wnt5a 3'UTR reporter plasmid was co-transfected into U87 and U251 cells with miR-375 mimics or related control. As shown in Figure 3B, U87 and U251 cells co-trans- fected with miR-375 mimics and wild-type Wnt5a 3'UTR reporter plasmid caused a significant decline in reporter activity $(p<0.05)$. Furthermore, we further detected the direct effect of miR-375 on Wnt5a expression. RT-PCR and Western blot analysis showed that the level of Wnt5a protein or mRNA expression was down-regulated by the miR-375 mimics in U87 and U251 cells (Figures 3C-3E). These data imply that Wnt5a is a direct target of miR-375 in GBM cells.

Wnt5a was involved in miR-375 induced proliferation and invasion in GBM cells. To confirm whether Wnt5a was involved in miR-375 induced proliferation and invasion in GBM cells, we performed a rescue assay by overexpressing Wnt5a. U87 and U251 cells were co-transfected with the miR-375 mimics and Wnt5a expressing vectors. Western blot

$\mathbf{A}$ miR-137 3 ' - A GGUGCGCUCGGCUUGC_U U U U U U - 5 '

Wnt5a 3'UTR (WT) 3 ' - U C U UA UA UAU G UAA U GGAAC A A - 5 '

Wnt5a 3'UTR (Mut) 3 ' - U C U U A U A UA U G UAA U G C U U G U U - 5 '

B

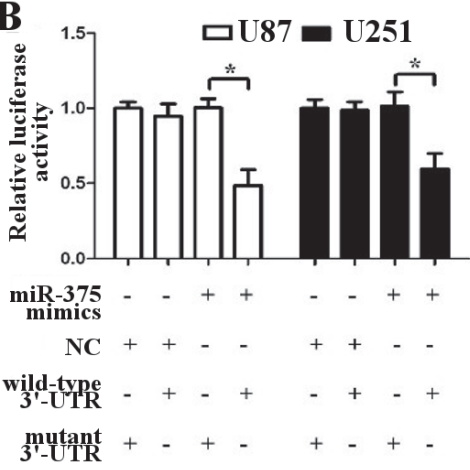

D
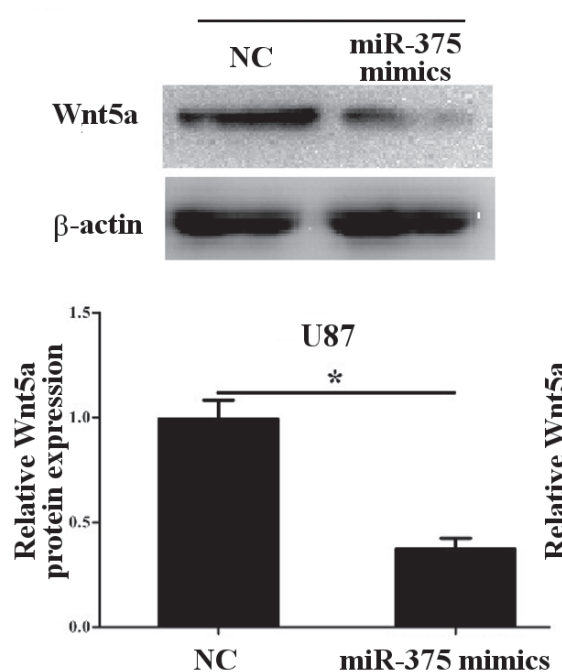

C

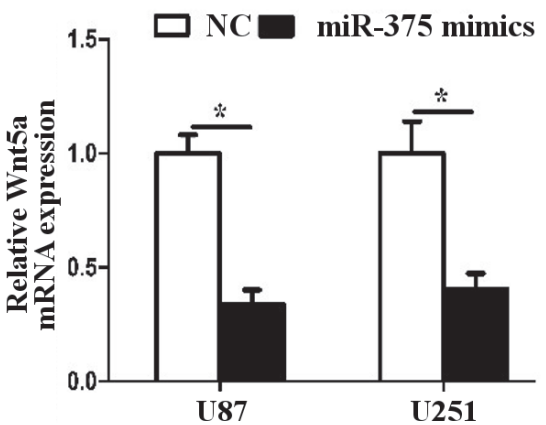

$\mathbf{E}$

$\mathbf{U} 251$
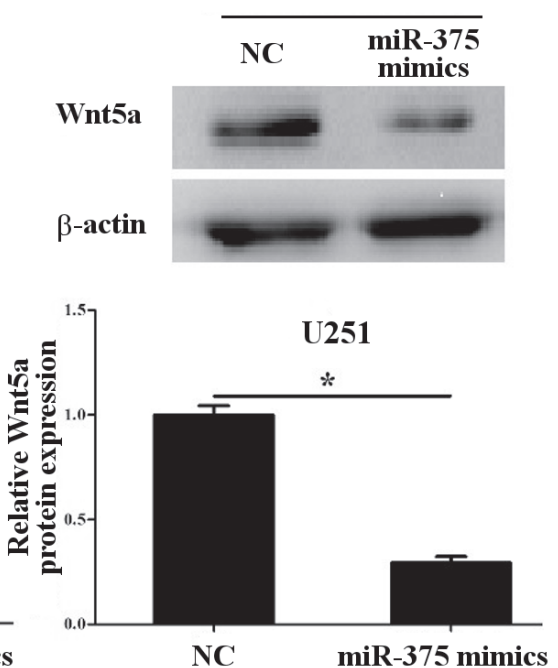

Figure 3. Wnt5a is direct target of miR-375 in GBM. A) Presentation of the miR-375 binding site within the 3'-UTR of Wnt5a. B) Luciferase reporter assay indicated that the inhibitory effect of miR-375 on Wnt5a involved binding to the 3'UTR in U87 and U251 cells. C) RT-PCR detected Wnt5a mRNA expression in U87 and U251 cells transfected with the miR-375 mimics and negative control (NC) for 24 h. D) Western blot detected Wnt5a protein expression in U87 and U251 cells transfected with the miR-375 mimics and negative control (NC) for $24 \mathbf{h} .{ }^{*} \mathbf{p}<0.05$. 

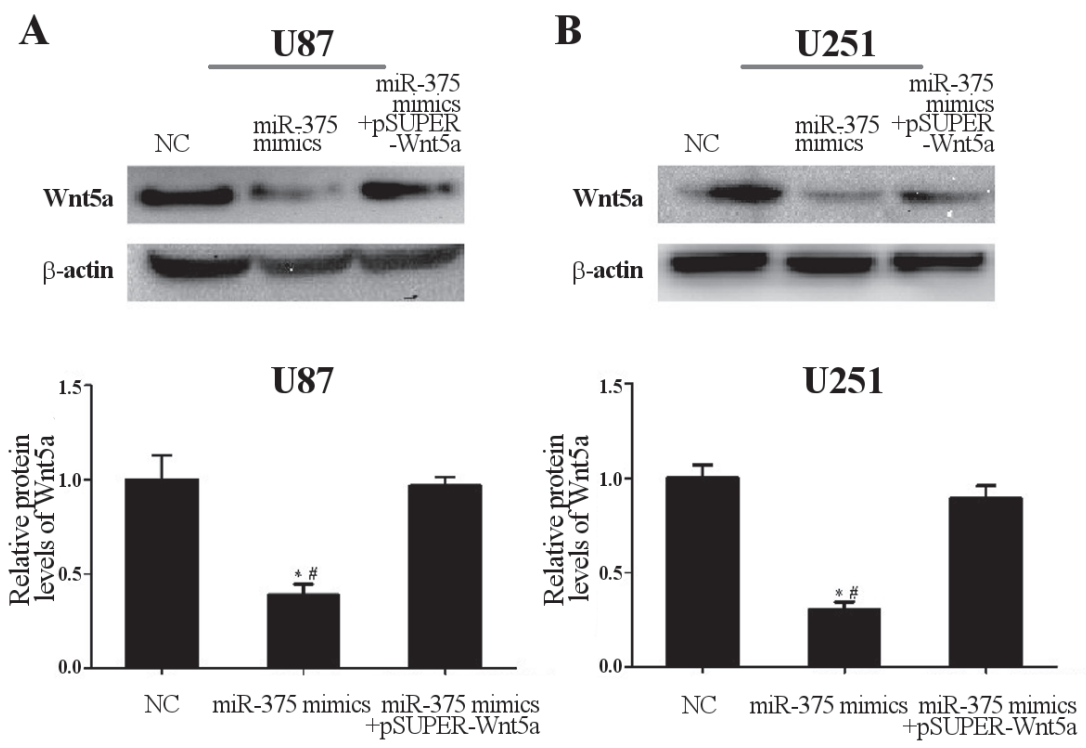

Figure 4. Western blot detected Wnt5a protein expression in U87 (A) and U251 (B) cells transfected with the miR-375 mimics with or without pSUPERWnt5a. ${ }^{\star} p<0.05$ vs negative control (NC), ${ }^{*} p<0.05$ vs miR-375 mimics + pSUPER-Wnt5a.

analysis confirmed that low expression of Wnt5a by miR-375 overexpression was rescued by Wnt5a expressing vector transfection in U87 and U251 cells (Figure 4). In addition, correlation analysis showed that miR-375 upregulationinduced proliferation and invasive abilities of the GBM cell lines were markedly reversed by Wnt5a overexpression (Figure 5). Our results suggest that Wnt5a acts as a miR-375 target and is involved in regulating the proliferation as well as invasion of GBM cells.

\section{Discussion}

Increasing evidence shows that miRNAs are important players in GBM development [13-15]. Abnormal expression of miRNAs is related to carcinogenesis of GBM, such as tumor apoptosis, metastasis, invasion, growth and so on. For instance, miR-375 has been reported to be significantly dysregulated in many tumors [11, 16, 17]. Low miR-375 expression was found in liver cancer by Xie et al [11]. Zehentmayr $\mathrm{F}$ et al found that miR-375 expression was down-regulated in breast cancer tissues and could be considered as a marker of breast cancer patients [17]. However, the role of miR-375 in GBM remains inadequately understood. In this study, we investigated the expression level of miR-375 in GBM and found that miR-375 was markedly down-regulated in GBM tissues compared to normal brain tissues. Further studies show that miR-375 is differentially down-regulated in GBM cell lines. Taken together, our research indicates that miR-375 might be a tumor suppressor in the progression of GBM.

To further test the miR-375 function in cell proliferation and invasion of GBM, we transfected U87 or U251 cells with
miR-375 mimics, miR-375 inhibitor or the relative miR-NC. Result of MTT, trans-well migration and invasion assays indicate that overexpression of miR-375 in U87 or U251 inhibits cell growth, migration as well as invasion. However, down-regulation of miR-375 had reverse effects on cell proliferation, migration and invasion. These findings suggest that miR-375 suppresses the GBM development.

Next, we studied the miR-375 regulatory mechanism in GBM and by bioinformatic algorithms found that Wnt5a was a miR-375 target gene. Wnt5a is a critical regulator of Wnt signaling pathway and is involved in cell apoptosis, proliferation, self-renewal and differentiation [18-20]. Evidence suggests that Wnt5a is highly expressed in many tumor types, such as prostate cancer, rectal cancer, osteosarcoma, and others $[19,20]$. For GBM, Wnt5a has been reported to possess tumor-suppressive function in GBM tissues [21]. Yu et al investigated the relationship between Wnt5a and proliferation of human glioblastoma cells [21]. They reported that down-regulation of Wnt5a expression as a result of RNA interference reduced proliferation in GBM-05 and U87MG cells in vitro and reduced tumorigenicity in vivo [21]. Recently, many researches have explored relative regulators which influence the Wnt5a expression. Wei R et al. identified Wnt5a as a direct target of miR-217 in osteosarcoma [22]. In their study, overexpressed Wnt5a could reverse miR-217inhibited migration, invasion and proliferation in osteosarcoma cells. In our study, luciferase assay results confirmed that miR-375 efficiently influenced luciferase expression through the 3'UTR of Wnt5a, and overexpression of Wnt5a could significantly reverse miR-375-mediated proliferation, migration and invasion in GBM cells. Our results illustrate that up-regulation of miR-375 in GBM cells may facilitate 

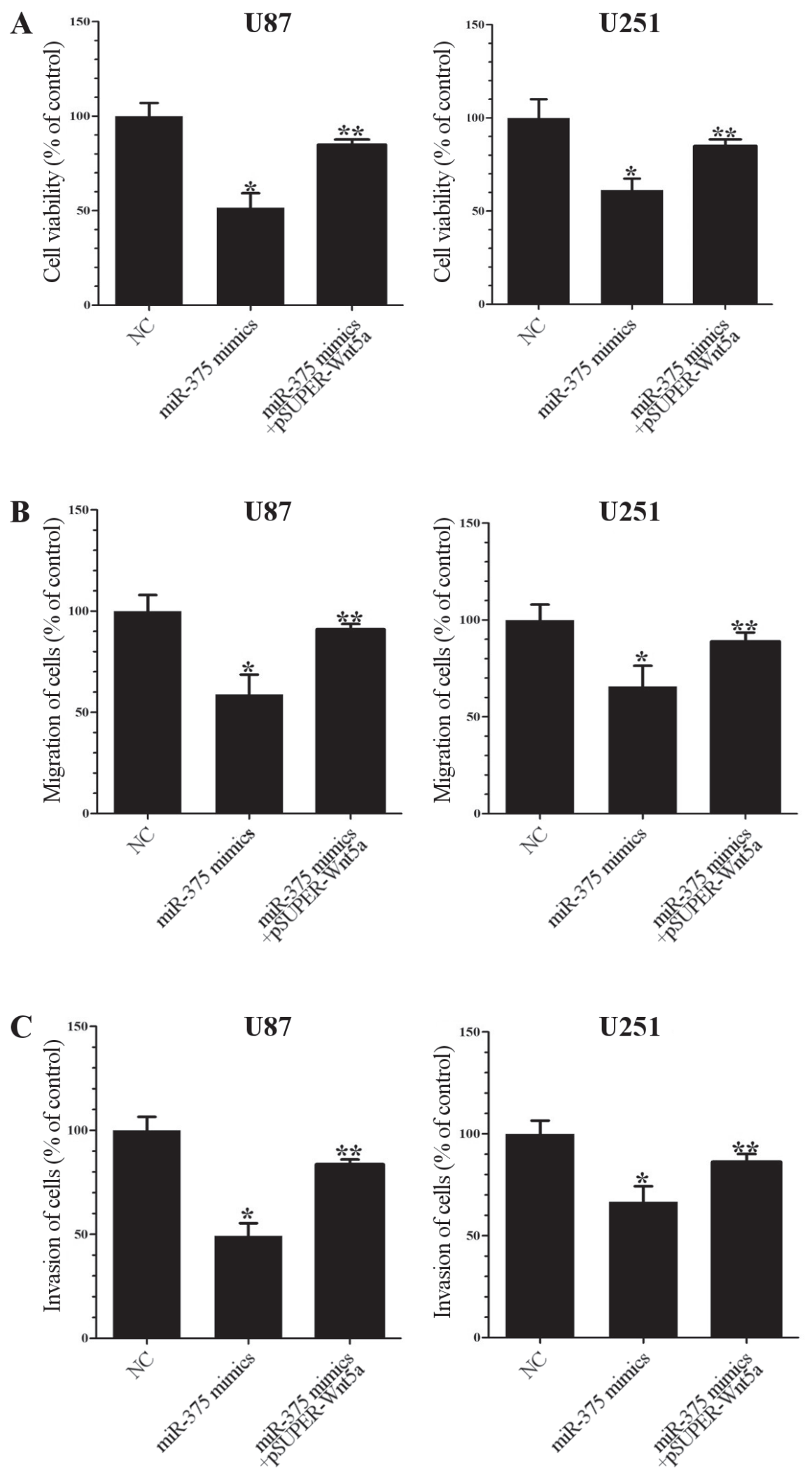

Figure 5. Overexpression of Wnt5a reverses the effect of miR-375 in GBM cells. Cell proliferation (A), migration (B) and invasion (C) capability in U87 and $\mathrm{U} 251$ cells were detected by the MTT assay and trans-well migration and invasion assay, respectively. ${ }^{\star} \mathrm{p}<0.05 \mathrm{vs}$ negative control (NC), ${ }^{\star *} \mathrm{p}<0.05$ vs miR-375 mimics. 
decreasing of the Wnt5a protein or mRNA expression level and in turn contribute to GBM development.

In summary, our results provide that miR- 375 is frequently down-regulated in clinical GBM tissues and cell lines and has an essential role in the regulation of GBM cell invasion and proliferation. We also identified that miR-375 exerted its functions partially by targeting Wnt5a in GBM. Our findings suggest that miR-375 might be a possible therapeutic agent for GBM.

Acknowledgements: This work is supported by Hai Yan Project of Harbin Medical University Cancer Hospital (No. JJQN2017-01)

\section{References}

[1] FULTON B, SHORT SC, JAMES A, NOWICKI S, MCBAIN C et al. PARADIGM-2: Two parallel phase I studies of olaparib and radiotherapy or olaparib and radiotherapy plus temozolomide in patients with newly diagnosed glioblastoma, with treatment stratified by MGMT status. Clin Transl Radiat Oncol 2018; 8: 12-16. https://doi.org/10.1016/j. ctro.2017.11.003

[2] CAI X, SUGHRUE ME. Glioblastoma: new therapeutic strategies to address cellular and genomic complexity. Oncotarget 2018; 9: 9540-9554. https://doi.org/10.18632/oncotarget.23476

[3] MUSCAT AM, WONG NC, DRUMMOND KJ, ALGAR EM, KHASRAW $\mathrm{M}$ et al. The evolutionary pattern of mutations in glioblastoma reveals therapy-mediated selection. Oncotarget 2018; 9: 7844-7858. https://doi.org/10.18632/ oncotarget.23541

[4] DAI Y, XIE CH, NEIS JP, FAN CY, VURAL E et al. MicroRNA expression profiles of head and neck squamous cell carcinoma with docetaxel-induced multidrug resistance. Head Neck 2011; 33: 786-791. https://doi.org/10.1002/hed.21540

[5] NARONHA V, PINNINTI R, JOSHI A, PRABHASH K. Chemotherapy for obstructive atelectasis in nonsmall cell lung cancer: Is this a treatment option. Indian J Cancer 2015; 52: 368-369. https://doi.org/10.4103/0019-509X.176756

[6] CHANG L, LEI X, QIN YU, ZHANG X, JIN H et al. MicroRNA-133b inhibits cell migration and invasion by targeting matrix metalloproteinase 14 in glioblastoma. Oncol Lett 2015; 10: 2781-2786. https://doi.org/10.3892/ol.2015.3657

[7] ROSCA A, ANTON G, RUTA S. MICRORNA BIOGENESIS AND ITS ROLE IN HIV-1 INFECTION. Roum Arch Microbiol Immunol 2014; 73: 84-91.

[8] HUANG Y, LU X, MA J. Toxicity of silver nanoparticles to human dermal fibroblasts on microRNA level. J Biomed Nanotechnol 2014; 10: 3304-3317.

[9] PATTERSON EE, HOLLOWAY AK, WENG J, FOJO T, KEBEBEW E. MicroRNA profiling of adrenocortical tumors reveals miR-483 as a marker of malignancy. Cancer 2011; 117: 1630-1639. https://doi.org/10.1002/cncr.25724
[10] OU J, KOU L, LIANG L, TANG C. MiR-375 attenuates injury of cerebral ischemia/reperfusion via targetting Ctgf. Biosci Rep 2017; 37. https://doi.org/10.1042/BSR20171242

[11] XIE D, YUAN P, WANG D, JIN H, CHEN H. Expression and prognostic significance of miR-375 and miR-221 in liver cancer. Oncol Lett 2017; 14: 2305-2309. https://doi.org/10.3892/ ol.2017.6423

[12] XUE H, YU Z, LIU Y, YUAN W, YANG T et al. Delivery of miR-375 and doxorubicin hydrochloride by lipid-coated hollow mesoporous silica nanoparticles to overcome multiple drug resistance in hepatocellular carcinoma. Int J Nanomedicine 2017; 12: 5271-5287. https://doi.org/10.2147/IJN. S135306

[13] LIU H, LIU N, CHENG Y, JIN W, ZHANG P et al. Hexokinase 2 (HK2), the tumor promoter in glioma, is downregulated by miR-218/Bmil pathway. PLoS One 2017; 12: e0189353. https://doi.org/10.1371/journal.pone.0189353

[14] CHENG Z, LI Z, MA K, LI X, TIAN N et al. Long Non-coding RNA XIST Promotes Glioma Tumorigenicity and Angiogenesis by Acting as a Molecular Sponge of miR-429. J Cancer 2017; 8: 4106-4116. https://doi.org/10.7150/jca.21024

[15] LIU T, WU C, WENG G, ZHAO Z, HE X et al. Bufalin Inhibits Cellular Proliferation and Cancer Stem CellLike Phenotypes via Upregulation of MiR-203 in Glioma. Cell Physiol Biochem 2017; 44: 671-681. https://doi. org/10.1159/000485279

[16] WANG Y, LIEBERMAN R, PAN J, ZHANG Q, DU M et al. miR-375 induces docetaxel resistance in prostate cancer by targeting SEC23A and YAP1. Mol Cancer 2016; 15: 70. https://doi.org/10.1186/s12943-016-0556-9

[17] ZEHENTMAYR F, HAUSER-KRONBERGER C, ZELLINGER B, HLUBEK F, SCHUSTER C et al. Hsa-miR-375 is a predictor of local control in early stage breast cancer. Clin Epigenetics 2016; 8: 28. https://doi.org/10.1186/s13148-0160198-1

[18] YAMAMOTO $\mathrm{H}$, OUE $\mathrm{N}$, SATO A, HASEGAWA $\mathrm{Y}$, YAMAMOTO $\mathrm{H}$ et al. Wnt5a signaling is involved in the aggressiveness of prostate cancer and expression of metalloproteinase. Oncogene 2010; 29: 2036-2046. https://doi. org/10.1038/onc.2009.496

[19] ENOMOTO M, HAYAKAWA S, ITSUKUSHIMA S, REN DY, MATSUO M et al. Autonomous regulation of osteosarcoma cell invasiveness by Wnt5a/Ror2 signaling. Oncogene 2009; 28: 3197-3208. https://doi.org/10.1038/onc.2009.175

[20] YAMAGATA K, LI X, IKEGAKI S, ONEYAMA C, OKADA $\mathrm{M}$ et al. Dissection of Wnt5a-Ror2 signaling leading to matrix metalloproteinase (MMP-13) expression. J Biol Chem 2012; 287: 1588-1599. https://doi.org/10.1074/jbc.M111.315127

[21] YU JM, JUN ES, JUNG JS, SUH SY, HAN JY et al. Role of Wnt5a in the proliferation of human glioblastoma cells. Cancer Lett 2007; 257: 172-181. https://doi.org/10.1016/j.canlet.2007.07.011

[22] WEI R, DENG Z, SU J. miR-217 targeting Wnt5a in osteosarcoma functions as a potential tumor suppressor. Biomed Pharmacother 2015; 72: 158-164. https://doi.org/10.1016/j. biopha.2015.04.012 